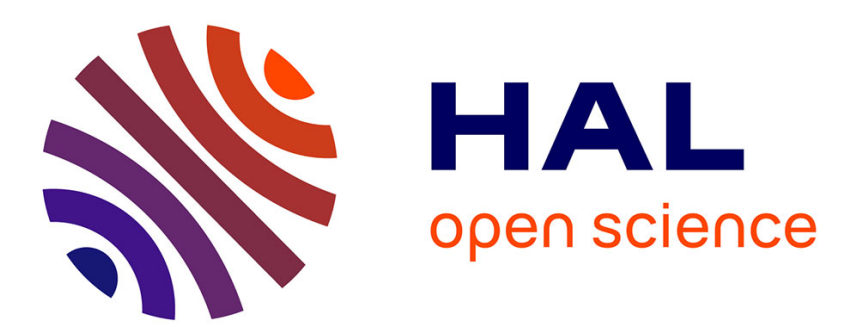

\title{
Elevation Beamforming in a Multi-Cell Full Dimension Massive MIMO System
}

Qurrat-Ul-Ain Nadeem, Abla Kammoun, Merouane Debbah, Mohamed-Slim

Alouini

\section{- To cite this version:}

Qurrat-Ul-Ain Nadeem, Abla Kammoun, Merouane Debbah, Mohamed-Slim Alouini. Elevation Beamforming in a Multi-Cell Full Dimension Massive MIMO System. 2018 IEEE Wireless Communications and Networking Conference (WCNC), Apr 2018, barcelone, Spain. 10.1109/WCNC.2018.8377262 . hal-01930953

\section{HAL Id: hal-01930953 \\ https://hal-centralesupelec.archives-ouvertes.fr/hal-01930953}

Submitted on 3 Jan 2019

HAL is a multi-disciplinary open access archive for the deposit and dissemination of scientific research documents, whether they are published or not. The documents may come from teaching and research institutions in France or abroad, or from public or private research centers.
L'archive ouverte pluridisciplinaire HAL, est destinée au dépôt et à la diffusion de documents scientifiques de niveau recherche, publiés ou non, émanant des établissements d'enseignement et de recherche français ou étrangers, des laboratoires publics ou privés. 


\title{
Elevation Beamforming in a Multi-Cell Full Dimension Massive MIMO System
}

\author{
Qurrat-Ul-Ain Nadeem*, Abla Kammoun*, Mérouane Debbah ${ }^{\dagger}$, and Mohamed-Slim Alouini* \\ * CEMSE Division, King Abdullah University of Science and Technology (KAUST), Thuwal, Saudi Arabia \\ Email: \{qurratulain.nadeem,abla.kammoun,slim.alouini\}@kaust.edu.sa \\ $\dagger$ Mathematical and Algorithmic Sciences Lab, Huawei France R\&D, Paris and CentraleSupélec, Gif-sur-Yvette, France \\ Email: merouane.debbah@huawei.com, merouane.debbah@centralesupelec.fr
}

\begin{abstract}
The 3GPP Release-13 has recently introduced fulldimension multiple-input multiple-output (FD-MIMO) technology as a practical way to deploy massive MIMO arrays within feasible base station (BS) form factors through the use of active antenna systems (AAS) with two-dimensional (2D) planar array structures. The 2D arrangement of antenna elements, where the elements in each antenna port are fed with downtilt weights, provides the additional ability of adaptive electronic beam control in the elevation dimension. This work focuses on the previously unaddressed problem of determining the optimal downtilt weight vectors for the antenna ports in each cell of a multicell multi-user system. The optimization criteria is to maximize the minimum signal-to-intra-cell-interference ratio within a cell while constraining the inter-cell interference leakage. A quasioptimal solution is obtained for the weight vectors through the application of semi-definite relaxation and Dinkelbach's method. The proposed algorithm is shown to perform better than the existing schemes even under the effects of pilot contamination.
\end{abstract}

\section{INTRODUCTION}

Massive multiple-input multiple-output (MIMO) technology is a promising candidate for performance enhancement in Fifth Generation (5G) communication systems [1]. One main challenge in the deployment of massive MIMO antenna arrays at base stations (BS)s operating at typical LTE carrier frequencies is the limited installation space at the top of BS towers. To address this challenge, the 3rd Generation Partnership Project (3GPP) Release-13 has proposed the use of FD-MIMO technology that utilizes an active antenna system (AAS) with a two-dimensional (2D) planar array structure [2].

FD-MIMO has two important distinguishing features. First, the number of antenna elements supported within feasible BS form factors has increased due to the use of $2 \mathrm{D}$ antenna arrays. Second, FD-MIMO provides the ability of adaptive electronic beam control in both the elevation and the traditional azimuth dimensions. The radio resource is organized on the basis of antenna ports, where each port consists of a number of physical antenna elements arranged vertically [3]. The vertical dimension of the antenna port radiation pattern can be adapted dynamically using the downtilt weights applied to the elements within it. This helps realize high resolution userspecific beamforming in three-dimensional (3D) space [4]-[6].

Several field trials [6], [7] and theoretical studies [8]-[10] have demonstrated the performance gains achievable through elevation beamforming. The authors in [6] adapt the vertical dimension of the antenna port radiation pattern at the BS individually for each user during field trials. The authors in [8] propose the management of inter-cell interference by coordinating the beamforming jointly in the azimuth and elevation planes. However, this work like the others on this topic [9], [10] use the approximate antenna port radiation pattern expressions from [11] to find the optimal downtilt angle and ignore the dependency of the pattern on the number of elements constituting the port, their relative positions and applied weights [2], [3]. This necessitates the development of practical elevation beamforming methods.

This paper proposes an elevation beamforming algorithm that optimizes the downtilt antenna port weight vectors applied at the BSs of a multi-cell massive MIMO system, utilizing the quasi-static channel covariance matrices of the users. The signal to interference ratio (SIR) based problem formulation is intractable in this setting. Alternatively, this work utilizes the concept of leakage, which refers to the interference caused by the signal intended for the user of interest to the remaining users [12], [13]. We first derive deterministic approximations for the SIR and inter-cell interference leakage that are tight in the asymptotic regime. Under the assumption that the antenna ports within a cell transmit using the same downtilt weight vector, we consider the problem of determining the optimal vector for each cell that maximizes the minimum signal-tointra-cell-interference ratio in that cell while constraining the interference leakage to the users in other cells. Semi-definite relaxation and Dinkelbach's method are used to obtain a quasi-optimal solution for the resulting fractional optimization problem. The proposed algorithm is shown to perform better than the existing schemes even under the effects of pilot contamination using simulations.

The rest of this article is organized as follows. Section II outlines the system model. Section III proposes the elevation beamforming algorithm for a multi-cell system. Section IV includes simulation results and section $\mathrm{V}$ concludes the article.

\section{SySTEM MOdeL}

This section introduces the $2 \mathrm{D}$ active antenna array, the $3 \mathrm{D}$ channel model and the spatial correlation function (SCF).

\section{A. Active Antenna Array for FD-MIMO}

The whole idea of FD-MIMO revolves around the use of an AAS with a 2D planar array structure in each cell. 


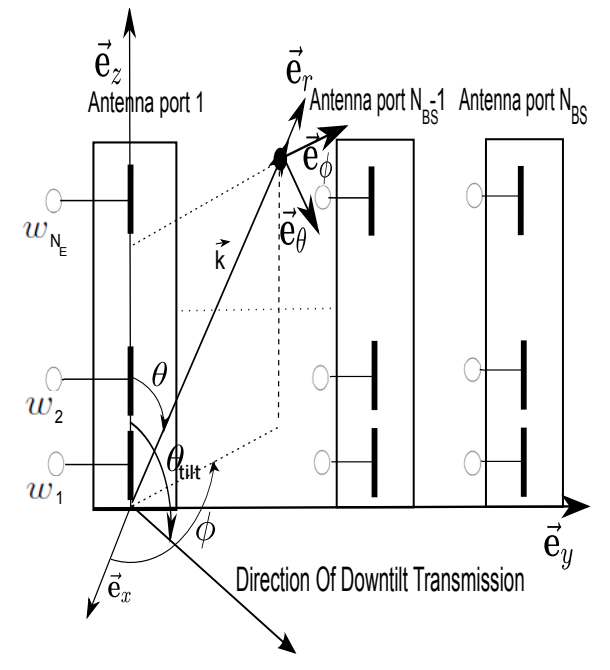

Fig. 1. Active antenna array deployed at each cell.

The AAS integrates the active transceiver unit array into the passive antenna element array, allowing the gain, beamwidth and downtilt angle of the transmit beam to be controlled adaptively by changing the phase of the antenna element excitation applied through the downtilt weights [4]. The 2D arrangement of active antenna elements allows for the control of the radio wave in the $3 \mathrm{D}$ space.

The radio resource is organized on the basis of antenna ports, where each port is mapped to $N_{E}$ physical antenna elements arranged vertically. The elements in a port carry the same signal and are fed with corresponding downtilt weights $w_{k}, k=1, \ldots, N_{E}$, to focus the wavefront in the targeted user's direction, referred to as the downtilt angle $\theta_{\text {tilt }}$. There are $N_{B S}$ such ports placed at equidistant positions in the $\hat{\mathbf{e}}_{y}$ direction. The resulting configuration for vertically polarized antenna elements is shown in Fig. 1.

\section{B. 3D Channel Model}

The existing 2D channel models are inadequate for the evaluation and design of FD-MIMO techniques, which require the development of 3D channel models. Unlike the existing approximate approach towards modeling the 3D radiation pattern of an antenna port [11], the 3GPP now proposes the use of the exact antenna port radiation pattern expression, which is a superposition of the element radiation pattern and the array factor for that port, where the element radiation pattern is given in the linear scale as $g_{E}(\phi, \theta)=g_{E, H}(\phi) g_{E, V}(\theta)$ with [14],

$$
\begin{aligned}
& g_{E, H}(\phi)=\exp \left(-1.2\left(\frac{\phi}{\phi_{3 d B}}\right)^{2} \ln 10\right), \\
& g_{E, V}(\theta)=\exp \left(-1.2\left(\frac{\theta-\frac{\pi}{2}}{\theta_{3 d B}}\right)^{2} \ln 10\right),
\end{aligned}
$$

where $\phi$ and $\theta$ denote the azimuth and elevation angle of departure (AoD) respectively, $g_{E, H}(\phi)$ and $g_{E, V}(\theta)$ are the radiation patterns in the horizontal and vertical directions respectively, and $\phi_{3 d B}$ and $\theta_{3 d B}$ are the half power beamwidths in the azimuth and elevation domains respectively.
The overall array radiation pattern is a function of this individual element radiation pattern and the array factor matrix, A, for the AAS given by [3],

$$
\mathbf{A}=\mathbf{W} \circ \mathbf{V},
$$

where $\circ$ is the Hadamard product, $\mathbf{V}$ is a $N_{E} \times N_{B S}$ array response matrix with each entry given by,

$$
\begin{aligned}
& {[\mathbf{V}]_{z, s}(\phi, \theta)=\exp \left(i 2 \pi \left((s-1) \frac{d_{y}}{\lambda} \sin \phi \sin \theta+(z-1)\right.\right.} \\
& \left.\left.\times \frac{d_{z}}{\lambda} \cos \theta\right)\right) \quad z=1, \ldots, N_{E}, \quad s=1, \ldots, N_{B S}
\end{aligned}
$$

where $d_{y}$ is the horizontal separation between the antenna ports and $d_{z}$ is the vertical separation between the antenna elements, with the phase reference at the origin.

Also $\mathbf{W}$ is a $N_{E} \times N_{B S}$ downtilt weight matrix, where the $s^{t h}$ column denoted as $\mathbf{w}_{s}$, represents the weight vector for the antenna port $s$. Denoting the $s^{t h}$ column of $\mathbf{V}$ as $\mathbf{v}_{s}\left(\phi_{n}, \theta_{n}\right)$, the $3 \mathrm{D}$ channel constituted by antenna port $s$ is given as,

$$
[\mathbf{h}]_{s}=\mathbf{w}_{s}^{T} \sum_{n=1}^{N} \alpha_{n} \sqrt{g_{E}\left(\phi_{n}, \theta_{n}\right)} \mathbf{v}_{s}\left(\phi_{n}, \theta_{n}\right),
$$

where $N$ is the number of propagation paths and $\alpha_{n} \sim$ i.i.d $\mathcal{C N}\left(0, \frac{1}{N}\right)$ is the amplitude of the $n^{\text {th }}$ path.

\section{Spatial Correlation Function for Antenna Ports}

It follows from (5) that the SCF for the channels constituted by antenna ports $s$ and $s^{\prime}$ will be given as,

$$
\begin{aligned}
& \rho\left(s, s^{\prime}\right)=\sum_{z=1}^{N_{E}} \sum_{z^{\prime}=1}^{N_{E}} w_{s}^{z} w_{s^{\prime}}^{z^{\prime} *} \mathbb{E}\left[g_{E}(\phi, \theta) v_{s}^{z}(\phi, \theta) v_{s^{\prime}}^{z^{\prime} *}(\phi, \theta)\right] \\
& =\sum_{z=1}^{N_{E}} \sum_{z^{\prime}=1}^{N_{E}} w_{s}^{z} w_{s^{\prime}}^{z^{\prime} *} \rho_{E}\left(s-s^{\prime}, z-z^{\prime}\right)
\end{aligned}
$$

where $w_{s}^{z}$ and $v_{s}^{z}(\phi, \theta)$ denote the $(z, s)^{t h}$ entries of $\mathbf{W}$ and $\mathbf{V}$ respectively, and $\rho_{E}\left(s-s^{\prime}, z-z^{\prime}\right)$ is the correlation between the channels constituted by $(z, s)^{t h}$ and $\left(z^{\prime}, s^{\prime}\right)^{t h}$ antenna elements in the AAS, where $z, z^{\prime}=1, \ldots, N_{E}$, $s, s^{\prime}=1, \ldots, N_{B S}$. The complete derivation of an analytical expression for $\rho_{E}\left(s-s^{\prime}, z-z^{\prime}\right)$ can be found in [14].

The $N_{B S} \times N_{B S}$ correlation matrix for the antenna ports constituting the AAS can therefore be written as [14],

$$
\mathbf{R}_{B S}=\widetilde{\mathbf{W}}^{H} \mathbf{R}^{E} \widetilde{\mathbf{W}},
$$

where $\widetilde{\mathbf{W}}$ is a $N_{B S} N_{E} \times N_{B S}$ block diagonal matrix of the weight vectors applied to the $N_{B S}$ antenna ports given as,

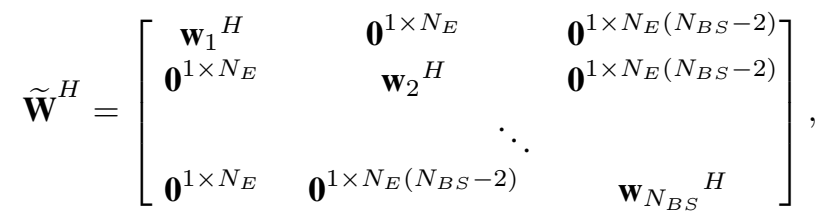

and $\mathbf{R}^{E}$ is the $N_{B S} N_{E} \times N_{B S} N_{E}$ correlation matrix for all the elements constituting the AAS defined as,

$$
\left[\mathbf{R}^{E}\right]_{\left(s^{\prime}-1\right) N_{E}+z^{\prime},(s-1) N_{E}+z}=\rho_{E}\left(s-s^{\prime}, z-z^{\prime}\right),
$$

where, $z, z^{\prime}=1, \ldots, N_{E}, \quad s, s^{\prime}=1, \ldots, N_{B S}$, and $\rho_{E}(s-$ $\left.s^{\prime}, z-z^{\prime}\right)$ is given by equation (28) in [14]. 


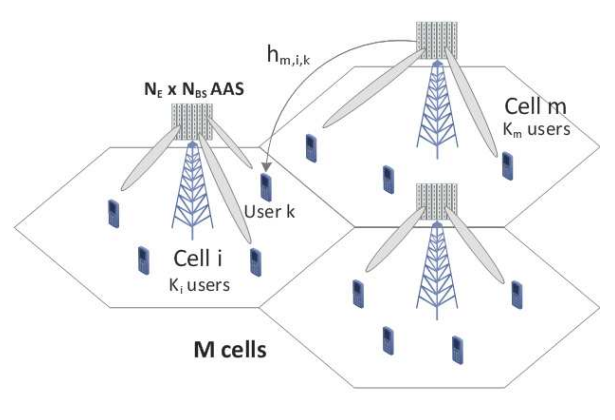

Fig. 2. Multi-cell layout.

\section{Elevation Beamforming Algorithm}

The performance of multi-cell systems is generally limited by inter-cell interference. In the presence of perfect channel state information (CSI), complete interference cancellation is possible by coordinated zero-forcing beamforming (ZFBF), but this also causes a loss in the energy of the desired signal.

Moreover, the CSI feedback overhead is prohibitively high when implementing ZFBF like fully digital precoding techniques in massive MIMO systems. In an attempt to reduce this overhead, the authors in [15] propose hybrid digital analog beamforming, where the analogue stage is implemented using channel statistics to reduce the channel dimension for the multi-user digital precoding stage. FD-MIMO AAS realizes this channel dimension reduction in the elevation beamforming stage using downtilt antenna port weight vectors that group antenna elements into a reduced number of antenna ports.

This work focuses on optimizing the downtilt antenna port weight vectors to maximize the minimum user signal to intracell interference ratio in each cell while constraining the interference leakage to the users in other cells [12], [13]. The problem is solved efficiently in the asymptotic regime, leveraging results from random matrix theory (RMT).

\section{A. Transmission Model}

Consider the downlink of a multi-cell system consisting of $M>1$ cells. Each cell has a central BS equipped with a $N_{E} \times N_{B S}$ AAS. Cell $m$ serves $K_{m}$ single-antenna users as shown in Fig. 2, where $m=1, \ldots, M$. We consider a time-division duplex (TDD) protocol in each cell. The received complex baseband signal $y_{i, k}$ at user $k$ in cell $i$ is given as,

$$
y_{i, k}=\sum_{m=1}^{M} \sqrt{\gamma_{m, i, k}} \mathbf{h}_{m, i, k}^{H} \mathbf{x}_{m}+n_{i, k},
$$

where $\mathbf{h}_{m, i, k}^{H} \in \mathbb{C}^{1 \times N_{B S}}$ is the downlink channel from BS $m$ to user $k$ in cell $i, \mathbf{x}_{m} \in \mathbb{C}^{N_{B S} \times 1}$ is the vector of transmit (Tx) signal from the BS $m$ and $n_{i, k} \sim \mathcal{C N}\left(0, \sigma^{2}\right)$ is the additive white Gaussian noise (AWGN) at user $k$ in cell $i$ with variance $\sigma^{2}$. Also $\gamma_{m, i, k}$ is the received signal power given as,

$$
\gamma_{m, i, k}=P_{T x, m} \times 10^{\frac{G_{E, \max }}{10}} \times \mathrm{PL}_{m, i, k} \times \mathrm{SF},
$$

where $P_{T x, m}$ is the average Tx power for cell $m, G_{E, \max }$ is the maximum antenna element gain, $\mathrm{PL}_{m, i, k}$ and $\mathrm{SF}$ denote the loss incurred in path loss and shadow fading respectively.
We consider Rayleigh correlated channel coefficients, where $\mathbf{h}_{m, i, k} \in \mathbb{C}^{N_{B S} \times 1}$ is modeled as,

$$
\mathbf{h}_{m, i, k}=\mathbf{R}_{B S_{m, i, k}}^{\frac{1}{2}} \mathbf{z}_{m, i, k},
$$

where $\mathbf{z}_{m, i, k} \sim \mathcal{C N}\left(\mathbf{0}, \mathbf{I}_{N_{B S}}\right)$ is the fast fading channel vector and $\mathbf{R}_{B S_{m, i, k}}$ is the channel covariance matrix of user $k$ in cell $i$ with respect to BS $m$ given by $\mathbf{R}_{B S_{m, i, k}}=\widetilde{\mathbf{W}}_{m}^{H} \mathbf{R}_{m, i, k}^{E} \widetilde{\mathbf{W}}_{m}$, where $\widetilde{\mathbf{W}}_{m}$ and $\mathbf{R}_{m, i, k}^{E}$ are defined in (8) and (9) respectively.

Assumption A-1. All channel covariance matrices have uniformly bounded spectral norm on $N_{B S}$, i.e.

$$
\lim \sup _{N_{B S}}\left\|\mathbf{R}_{B S_{m, i, k}}\right\|<+\infty, \quad \forall m, i, k .
$$

All BSs use linear precoding in the digital domain to mitigate inter-user interference. The precoding vector and the data symbol for user $k$ in cell $m$ are denoted by $\mathbf{g}_{m, k} \in \mathbb{C}^{N_{B S} \times 1}$ and $s_{m, k} \sim \mathcal{C N}(0,1)$ respectively. Consequently,

$$
\mathbf{x}_{m}=\sum_{k=1}^{K_{m}} \mathbf{g}_{m, k} s_{m, k}=\mathbf{G}_{m} \mathbf{s}_{m}
$$

where $\mathbf{G}_{m}$ is the $N_{B S} \times K_{m}$ precoding matrix and $\mathbf{s}_{m}$ is the $K_{m} \times 1$ vector of data symbols for cell $m$.

The computational complexity of the asymptotically optimal ZFBF scheme is prohibitively high in the large $\left(N_{B S}, K\right)$ regime, which motivates the use of maximum ratio transmission (MRT) in massive MIMO settings due to its low computational complexity, robustness, and high asymptotic performance [1]. The MRT precoding vector is given as,

$$
\mathbf{g}_{m, k}=\beta_{m} \mathbf{h}_{m, m, k},
$$

where $\beta_{m}$ is chosen to satisfy the total Tx power constraint. Since $P_{T x, m}$ is already included in (11), so the constraint is given as $\operatorname{tr}\left(\mathbf{G}_{m} \mathbf{G}_{m}^{H}\right)=1$ resulting in,

$$
\beta_{m}=\frac{1}{\sqrt{\operatorname{tr}\left(\mathbf{H}_{m, m} \mathbf{H}_{m, m}^{H}\right)}},
$$

where $\mathbf{H}_{m, m}=\left[\mathbf{h}_{m, m, 1} \mathbf{h}_{m, m, 2} \ldots \mathbf{h}_{m, m, K_{m}}\right] \in \mathbb{C}^{N_{B S} \times K_{m}}$.

\section{B. Problem Formulation}

The received signal $y_{i, k}$ in (10) is first decomposed to distinguish the desired signal from the interference as,

$$
\begin{gathered}
y_{i, k}=\sqrt{\gamma_{i, i, k}} \mathbf{h}_{i, i, k}^{H} \mathbf{g}_{i, k} s_{i, k}+\sum_{\substack{l=1 \\
l \neq k}}^{K_{i}} \sqrt{\gamma_{i, i, k}} \mathbf{h}_{i, i, k}^{H} \mathbf{g}_{i, l} s_{i, l} \\
+\sum_{\substack{m=1 \\
m \neq i}}^{M} \sum_{k^{\prime}=1}^{K_{m}} \sqrt{\gamma_{m, i, k}} \mathbf{h}_{m, i, k}^{H} \mathbf{g}_{m, k^{\prime}} s_{m, k^{\prime}}+n_{i, k} .
\end{gathered}
$$

The SIR at user $k$ in cell $i$ is now defined as,

$$
S I R_{i, k}=\frac{S_{i, k}}{I_{i, k}^{\text {intra }}+\sum_{\substack{m=1 \\ m \neq i}}^{M} I_{i, k}^{\text {inter }, m}},
$$

where $S_{i, k}=\gamma_{i, i, k}\left|\mathbf{h}_{i, i, k}^{H} \mathbf{g}_{i, k}\right|^{2}$ is the received signal power at user $k$ in cell $i, I_{i, k}^{\text {intra }}=\sum_{\substack{l=1 \\ l \neq k}}^{K_{i}} \gamma_{i, i, k}\left|\mathbf{h}_{i, i, k}^{H} \mathbf{g}_{i, l}\right|^{2}$ is the received intra-cell interference at user $k$ in cell $i$ and $I_{i, k}^{\text {inter, } m}=$ 


$$
\begin{aligned}
& S I R_{i, k}-\frac{\frac{1}{N_{B S}^{2}} \frac{\gamma_{i, i, k}}{\sum_{k=1}^{K_{i}} \operatorname{tr} \mathbf{R}_{B S i, i, k}}\left(\operatorname{tr} \mathbf{R}_{B S i, i, k}\right)^{2}}{\frac{1}{N_{B S}^{2}} \frac{\gamma_{i, i, k}}{\sum_{k=1}^{K_{i}} \operatorname{tr} \mathbf{R}_{B S i, i, k}} \sum_{l \neq k}^{K_{i}} \operatorname{tr}\left(\mathbf{R}_{B S i, i, k} \mathbf{R}_{B S i, i, l}\right)+\frac{1}{N_{B S}^{2}} \sum_{\substack{m=1 \\
m \neq i}}^{M} \frac{\gamma_{m, i, k}}{\sum_{k^{\prime}=1}^{K m} \operatorname{tr} \mathbf{R}_{B S m, m, k^{\prime}}} \sum_{k^{\prime}=1}^{K_{m}} \operatorname{tr}\left(\mathbf{R}_{B S m, i, k} \mathbf{R}_{B S m, m, k^{\prime}}\right)} \stackrel{a . s .}{\longrightarrow} 0, \\
& \overline{S I R}_{i, k}-\frac{\left(\frac{1}{N_{B S}} \sum_{s=1}^{N_{B S}} \mathbf{w}_{i, s}^{H} \mathbf{R}_{i, i, k, s s}^{E} \mathbf{w}_{i, s}\right)^{2}}{\frac{1}{N_{B S}^{2}} \sum_{l \neq k}^{K_{i}} \sum_{s, s^{\prime}=1}^{N_{B S}} \mathbf{w}_{i, s}^{H} \mathbf{R}_{i, i, k, s s^{\prime}}^{E} \mathbf{w}_{i, s^{\prime}} \mathbf{w}_{i, s^{\prime}}^{H} \mathbf{R}_{i, i, l, s^{\prime} s}^{E} \mathbf{w}_{i, s}} \stackrel{a . s .}{\longrightarrow} 0, \\
& L_{i, k}^{\text {inter }, m, k^{\prime}}-\gamma_{i, m, k^{\prime}} \frac{\sum_{s, s^{\prime}=1}^{N_{B S}} \mathbf{w}_{i, s}^{H} \mathbf{R}_{i, m, k^{\prime}, s s^{\prime}}^{E} \mathbf{w}_{i, s^{\prime}} \mathbf{w}_{i, s^{\prime}}^{H} \mathbf{R}_{i, i, k, s^{\prime} s}^{E} \mathbf{w}_{i, s}}{\sum_{k=1}^{K_{i}} \sum_{s=1}^{N_{B S}} \mathbf{w}_{i, s}^{H} \mathbf{R}_{i, i, k, s s}^{E} \mathbf{w}_{i, s}} \stackrel{a . s .}{\longrightarrow} 0,
\end{aligned}
$$

$\sum_{k^{\prime}=1}^{K_{m}} \gamma_{m, i, k}\left|\mathbf{h}_{m, i, k}^{H} \mathbf{g}_{m, k^{\prime}}\right|^{2}$ is the interference caused by cell $m$ to user $k$ in cell $i$. An asymptotic analysis of these terms would yield a deterministic approximation for the SIR in the large $\left(N_{B S}, N_{E}\right)$ regime as stated in the following theorem.

Theorem 1. Consider a multi-cell system where the BS in cell $i$ is equipped with a $N_{E} \times N_{B S}$ AAS that serves $K_{i}$ singleantenna users. Then in the large $\left(N_{B S}, N_{E}\right)$ regime and under Assumption A-1 and MRT precoding, $S I R_{i, k}$ converges as shown in (19), for $k=1, \ldots, K_{i}, i=1, \ldots, M$.

Proof. The deterministic equivalents of $S_{i, k}, I_{i, k}^{\text {intra }}$ and $I_{i, k}^{\text {inter, } m}$ are worked out separately using the fact that $\mathbf{h}_{i, i, k}=$

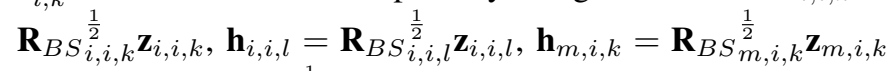
and $\mathbf{h}_{m, m, k^{\prime}}=\mathbf{R}_{B S_{m, m, k^{\prime}}} \mathbf{z}_{m, m, k^{\prime}}^{\frac{1}{2}}$ using (12) and applying the trace lemma [Lemma 14.2 [16]] repeatedly to the quadratic terms in $\mathbf{z} \sim \mathcal{C N}\left(\mathbf{0}, \mathbf{I}_{N_{B S}}\right)$.

Treating the SIR in (19) directly as an objective function of the weight vector optimization problem will render it NP-hard. To create a good balance between the interference and signal, we instead optimize the downtilt antenna port weight vectors for each cell with the objective of maximizing the minimum user signal to intra-cell interference ratio in that cell, denoted by $\overline{S I R}$, subject to constraints on the interference leakage caused to the users in other cells defined as [12], [13],

$$
L_{i, k}^{i n t e r, m, k^{\prime}}=\gamma_{i, m, k^{\prime}}\left|\mathbf{h}_{i, m, k^{\prime}}^{H} \mathbf{g}_{i, k}\right|^{2} .
$$

$L_{i, k}^{\text {inter, } m, k^{\prime}}$ represents the interference leakage caused by the communication between BS $i$ and user $k$ to user $k^{\prime}$ in cell $m$. An asymptotic analysis of (20) would yield a deterministic approximation for it in the large $\left(N_{B S}, N_{E}\right)$ regime as,

$$
L_{i, k}^{i n t e r, m, k^{\prime}}-\gamma_{i, m, k^{\prime}} \frac{\operatorname{tr}\left(\mathbf{R}_{B S i, m, k^{\prime}} \mathbf{R}_{B S i, i, k}\right)}{\sum_{k=1}^{K_{i}} \operatorname{tr}\left(\mathbf{R}_{B S i, i, k}\right)} \stackrel{a . s .}{\longrightarrow} 0
$$

where $k=1, \ldots, K_{i}, i=1, \ldots, M, k^{\prime}=1, \ldots, K_{m}$, $\underset{m \neq i}{m}=1, \ldots, M$. Now given that $\mathbf{R}_{B S_{m, i, k}}=\widetilde{\mathbf{W}}_{m}^{H} \mathbf{R}_{m, i, k}^{E} \widetilde{\mathbf{W}}_{m}$ as defined in (7), (8), and (9), the $\overline{S I R}_{i, k}$ and $L_{i, k}^{i n t e r, m, k^{\prime}}$ converge to (22) and (23) respectively, where $\mathbf{w}_{i, s}$ and $\mathbf{w}_{i, s^{\prime}}$ are the $N_{E} \times 1$ weight vectors for the antenna ports $s$ and $s^{\prime}$ at BS $i$ that form the block diagonal matrix $\widetilde{\mathbf{W}}_{i}$ in (8), $\mathbf{R}_{i, i, k, s s}^{E}$ is a $N_{E} \times N_{E}$ matrix given by $\mathbf{R}_{i, i, k}^{E}\left(\left[(s-1) N_{E}+1\right.\right.$ : $\left.\left.s N_{E}\right],\left[(s-1) N_{E}+1: s N_{E}\right]\right)$, where $\mathbf{R}_{i, i, k}^{E}$ is defined in (9). Similarly $\mathbf{R}_{i, i, k, s s^{\prime}}^{E}$ is a $N_{E} \times N_{E}$ matrix given by $\mathbf{R}_{i, i, k}^{E}\left(\left[(s-1) N_{E}+1: s N_{E}\right],\left[\left(s^{\prime}-1\right) N_{E}+1: s^{\prime} N_{E}\right]\right)$.

The problem of finding the optimal downtilt weight vectors for the antenna ports in cell $i, i=1, \ldots M$, is formulated as, Problem (P1):

$$
\begin{array}{ll}
\underset{\mathbf{w}_{i, 1}, \ldots \mathbf{w}_{i, N_{B S}}}{\operatorname{maximize}} & \underset{k \in\left\{1, \ldots, K_{i}\right\}}{\operatorname{minimize}} \overline{S I R}_{i, k} \\
\text { subject to } & \left\|\mathbf{w}_{i, s}\right\|_{2}=1, \quad s=1, \ldots, N_{B S}, \\
& \left|L_{i, k}^{\text {inter }, m, k^{\prime}}\right|<\epsilon, k^{\prime} \in\left\{1, \ldots, K_{m}\right\}, \\
& m_{m \neq i} \in\{1, \ldots, M\}, k \in\left\{1, \ldots, K_{i}\right\},
\end{array}
$$

where $\overline{S I R}_{i, k}$ and $L_{i, k}^{\text {inter, } m, k^{\prime}}$ are given by their deterministic equivalents in (22) and (23) respectively.

The problem is hard to solve because it considers the joint optimization of different weight vectors for different antenna ports in each cell. Even works that consider joint max-min problem formulations [17] with same beamforming weight vectors have shown the problem to be NP-hard and resort to semi-definite relaxation (SDR) to reach a tractable problem formulation. To this end, we make the following assumption.

Assumption A-2. All antenna ports in a cell transmit using the same optimal downtilt weight vector.

This scenario is referred to as multi-cell single downtilt beamforming (SDB). The optimization of the weight vectors for the active antenna array proposed in [3] is an unaddressed problem so it is useful to lay the groundwork and see the extent of performance gains realizable. Dropping the subscripts $s$ and $s^{\prime}$ in Problem $(P l)$ and substituting the positive semi-definite rank-one matrix $\mathbf{w}_{i} \mathbf{w}_{i}^{H} \in \mathbb{C}^{N_{E} \times N_{E}}$ for a positive semi-definite matrix $\mathbf{W}_{i} \in \mathbb{C}^{N_{E} \times N_{E}}$ of arbitrary rank, the semi-definite relaxed formulation of $P 1$ is given as,

Problem (P2):

$$
\max _{\mathbf{W}_{i}} \min _{k \in\left\{1, \ldots, K_{i}\right\}} \frac{\left(\sum_{s=1}^{N_{B S}} \operatorname{tr}\left(\mathbf{W}_{i} \mathbf{R}_{i, i, k, s s}^{E}\right)\right)^{2}}{\sum_{l \neq k}^{K_{i}} \sum_{s, s^{\prime}=1}^{N_{B S}} \operatorname{tr}\left(\mathbf{W}_{i} \mathbf{R}_{i, i, k, s s^{\prime}}^{E} \mathbf{W}_{i} \mathbf{R}_{i, i, l, s^{\prime} s}^{E}\right)}
$$

subject to $\mathbf{W}_{i} \succeq 0, \quad \operatorname{tr}\left(\mathbf{W}_{i}\right)=1$,

$$
\begin{gathered}
\gamma_{i, m, k^{\prime}} \sum_{s, s^{\prime}=1}^{N_{B S}} \operatorname{tr}\left(\mathbf{W}_{i} \mathbf{R}_{i, m, k^{\prime}, s s^{\prime}}^{E} \mathbf{W}_{i} \mathbf{R}_{i, i, k, s^{\prime} s}^{E}\right)-\epsilon \sum_{k}^{K_{i}} \sum_{s=1}^{N_{B S}} \operatorname{tr}\left(\mathbf{W}_{i}\right. \\
\left.\mathbf{R}_{i, i, k, s s}^{E}\right)<0, \quad k^{\prime} \in\left\{1, \ldots, K_{m}\right\}, m_{m \neq i} \in\{1, \ldots, M\}, \text { (29) }
\end{gathered}
$$




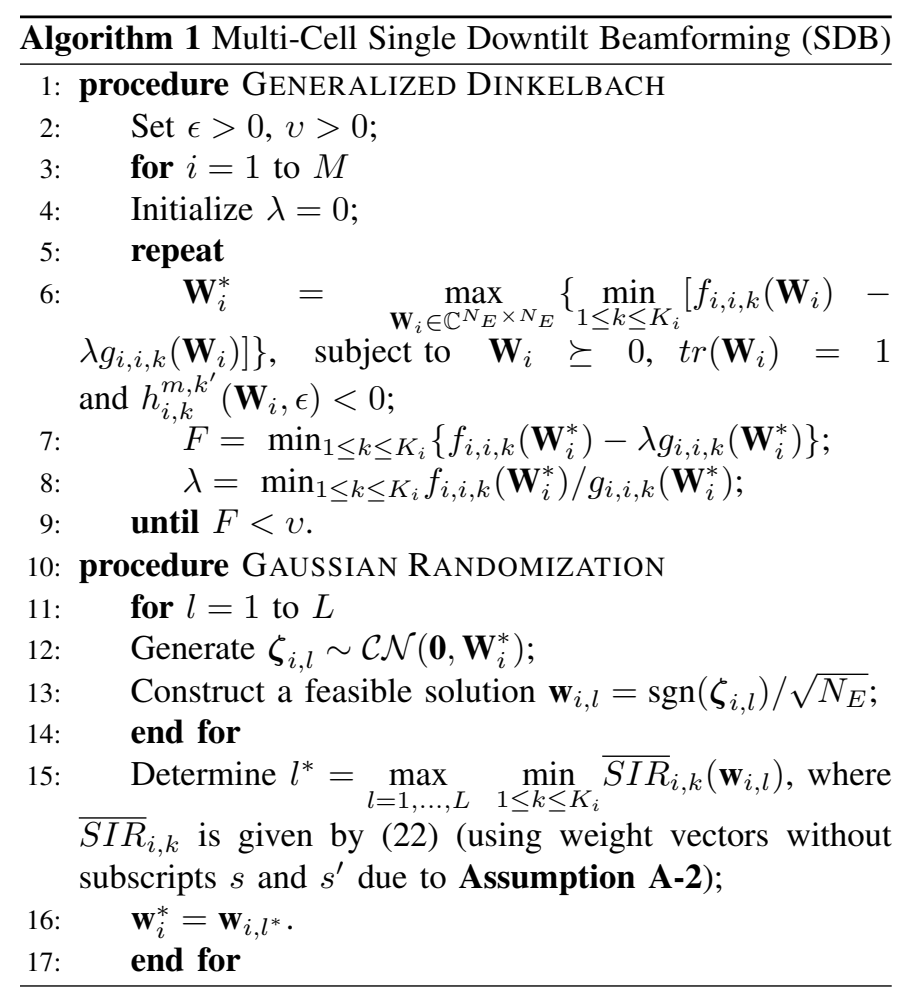

$k \in\left\{1, \ldots, K_{i}\right\}$. Problem (P2) is efficiently solved using fractional programmings tools as discussed now.

\section{Optimization Technique and Solution}

Fractional programming provides efficient tools to maximize the minimum of ratios in which the numerator is a concave function, the denominator is a convex function, and the constraint set is convex. An efficient method to do so is the generalized Dinkelbach's algorithm [18]. In order to apply this method, we reformulate Problem (P2) using the properties of vec function as [19],

$$
\begin{gathered}
\text { Problem (P3): } \max _{\mathbf{W}_{i}} \min _{k \in\left\{1, \ldots, K_{i}\right\}} \\
\frac{\sum_{s=1}^{N_{B S}} \operatorname{vec}\left(\mathbf{W}_{i}^{T}\right)^{T} \operatorname{vec}\left(\mathbf{R}_{i, i, k, s s}^{E}\right)}{\left(\operatorname{vec}\left(\mathbf{W}_{i}^{T}\right)^{T}\left(\sum_{l \neq k}^{K_{i}} \sum_{s, s^{\prime}=1}^{N_{B S}} \mathbf{R}_{i, i, l, s^{\prime} s}^{E} T^{T} \otimes \mathbf{R}_{i, i, k, s s^{\prime}}^{E}\right) \operatorname{vec}\left(\mathbf{W}_{i}\right)\right)^{\frac{1}{2}}}
\end{gathered}
$$

subject to $\mathbf{W}_{i} \succeq 0, \operatorname{tr}\left(\mathbf{W}_{i}\right)=1$,

$$
\begin{aligned}
& \gamma_{i, m, k^{\prime}} \operatorname{vec}\left(\mathbf{W}_{i}^{T}\right)^{T}\left(\sum_{s, s^{\prime}=1}^{N_{B S}} \mathbf{R}_{i, i, k, s^{\prime} s}^{E}{ }^{T} \otimes \mathbf{R}_{i, m, k^{\prime}, s s^{\prime}}^{E}\right) \operatorname{vec}\left(\mathbf{W}_{i}\right) \\
& -\epsilon \sum_{k}^{K_{i}} \sum_{s=1}^{N_{B S}} \operatorname{vec}\left(\mathbf{W}_{i}^{T}\right)^{T} \operatorname{vec}\left(\mathbf{R}_{i, i, k, s s}^{E}\right)<0 \\
& k^{\prime} \in\left\{1, \ldots, K_{m}\right\}, m_{m \neq i} \in\{1, \ldots, M\}, k \in\left\{1, \ldots, K_{i}\right\} .
\end{aligned}
$$

We denote the numerator in (30) by $f_{i, i, k}\left(\mathbf{W}_{i}\right)$ and the denominator by $g_{i, i, k}\left(\mathbf{W}_{i}\right), k=1, \ldots, K_{i}$, and the constraint in (32) as $h_{i, k}^{m, k^{\prime}}\left(\mathbf{W}_{i}, \epsilon\right)<0$.

It can be seen from (30) that $f_{i, i, k}\left(\mathbf{W}_{i}\right)$ is a linear function of $\mathbf{W}_{i}$. Also $g_{i, i, k}\left(\mathbf{W}_{i}\right)$ and $h_{i, k}^{m, k^{\prime}}\left(\mathbf{W}_{i}, \epsilon\right)$ are convex

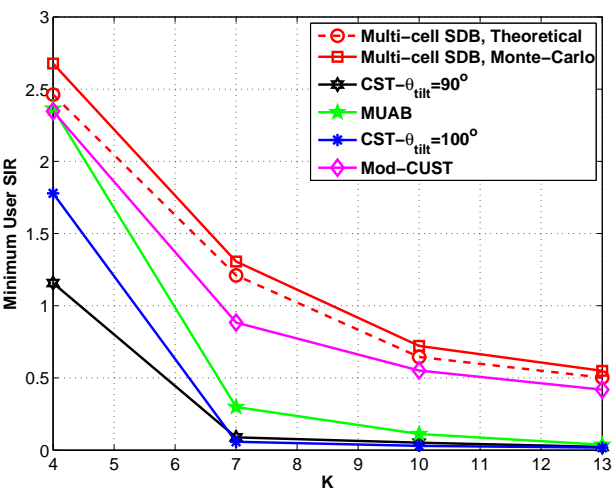

Fig. 3. Performance of SDB in a multi-cell system.

functions. Problem (P3) therefore considers a set of ratios $\left\{\frac{f_{i, i, k}\left(\mathbf{W}_{i}\right)}{g_{i, i, k}\left(\mathbf{W}_{i}\right)}\right\}_{k=1}^{K_{i}}$, where each ratio has an affine numerator, convex denominator and convex constraints and can therefore be solved using the generalized Dinkelbach's algorithm [18]. The Dinkelbach's procedure to solve Problem (P3) is formulated in Algorithm 1. Once the optimal $\mathbf{W}_{i}^{*}$ is obtained for $i=1, \ldots, M$ cells, the corresponding optimal weight vector $\mathbf{w}_{i}^{*}$ needs to be extracted. Generally the globally optimal solution $\mathbf{W}_{i}^{*}$ of Problem (P3) has a rank greater than one and therefore yields a quasi-optimal solution for Problem $(P 1)$. Besides the eigenvector approximation method, Gaussian randomization is another technique used in literature to extract an approximate solution from the SDR solution $\mathbf{W}_{i}^{*}$, that has good theoretical accuracy [17]. The idea is to generate a random vector $\boldsymbol{\zeta}_{i} \in \mathbb{C}^{N_{E} \times 1} \sim \mathcal{C N}\left(\mathbf{0}, \mathbf{W}_{i}^{*}\right)$ and use it to construct an approximate solution to Problem (P1). The specific design of the randomization procedure is problemdependent and is summarized at the end of Algorithm 1.

\section{Simulation Results}

The performance gains realizable through the proposed SDB algorithm are now studied using simulations. The elevation angles are generated according to Laplacian density spectrum, with mean $\operatorname{AoD} \theta_{0}$ and spread $\sigma_{t}$. The azimuth angles are generated using Von Mises distribution, with mean $\mu$ and a measure of spread, $\kappa_{t}$. The parameter values are set as $\theta_{3 d B}, \phi_{3 d B}=65^{\circ}, \sigma_{t}=10^{\circ}, \kappa_{t}=10, \mu=0, P_{T x, m}=20 \mathrm{~dB}$ $\forall m$ and $G_{E, \max }=8 \mathrm{dBi}$. The path loss expression and SF value are taken from [3] for the 3D urban macro scenario.

The system comprises of three neighboring hexagonal cells with an equal number of users, $K$, placed randomly in each cell at a minimum distance of $30 \mathrm{~m}$ from the BS. A $10 \times 14$ AAS is employed at the BS in each cell. The quasioptimal weight vector $\mathbf{w}_{i}^{*}$ for the antenna ports in each of the $i=1, \ldots, M$ cells is obtained using the SDB Algorithm 1, utilizing the users' channel covariance matrices computed using (7). The Monte-Carlo simulated minimum user SIR in (18) is then computed for each cell along with the deterministic equivalent in (19), under Assumptions A-1 and A-2. The result in Fig. 3 shows the minimum user SIR of the system. 


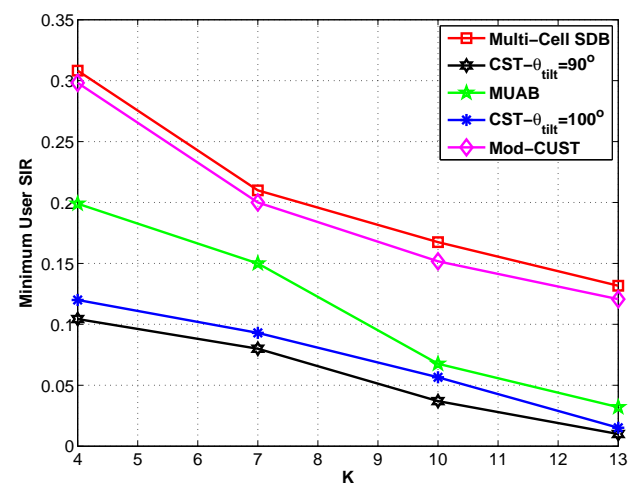

Fig. 4. Performance of SDB under pilot contamination for $\rho_{t r}=6 \mathrm{~dB}$.

Using a moderate number of antennas, a good match is obtained between the Monte-Carlo simulated SIR and its deterministic equivalent in Theorem 1 derived for the multicell SDB scenario. The performance of multi-cell SDB is compared to that of the following downtilting strategies.

1) Cell specific tilting (CST) [8] - A common fixed tilt, $\theta_{t i l t}$, is applied to the antenna ports at all BSs, with the weight vector computed using equation (9) in [14].

2) Coordinated user-specific tilting (CUST) [8] - A greedy tilt selection algorithm is utilized. We use (24) as the performance metric in the algorithm to allow for a fair comparison, and refer to the strategy as Mod-CUST.

3) Multi-user active beamforming (MUAB) [9] - The downtilt angle $\theta_{t i l t, i}$ for the antenna ports in cell $i$ is computed as the mean of the elevation line of sight angles of the users in cell $i, i=1, \ldots, M$.

All tilting strategies are simulated with MRT precoding in the horizontal domain. The proposed SDB scenario achieves significant performance gains as compared to existing elevation beamforming schemes as seen in Fig. 3. All existing schemes use the approximate antenna port radiation pattern expression from [11], limiting their use in actual FD-MIMO settings.

We also test the performance of our algorithm in the scenario where pilot contamination from adjacent cells corrupts the channel estimates used for MRT precoding. The MMSE estimate $\hat{\mathbf{h}}_{i, i, k}$ of $\mathbf{h}_{i, i, k}$ is given as $\mathbf{R}_{B S_{i, i, k}} \mathbf{Q}_{i, k}\left(\sum_{m=1}^{M} \mathbf{h}_{i, m, k}+\frac{1}{\sqrt{\rho_{t r}}} \mathbf{n}_{i, k}^{t r}\right)$, where $\mathbf{n}_{i, k}^{t r} \sim$ $\mathcal{C N}\left(\mathbf{0}, \mathbf{I}_{N_{B S}}\right)$ and $\rho_{t r}$ is the effective training SNR. Also $\mathbf{Q}_{i, k}=\left(\sum_{m=1}^{M} \mathbf{R}_{B S_{i, m, k}}+\frac{1}{\rho_{t r}} \mathbf{I}_{N_{B S}}\right)^{-1}$. The result in Fig. 4 shows that even under the effects of pilot contamination, the SDB algorithm performs better than the existing schemes.

\section{CONCLUSION}

This paper studied the architecture of the 2D active antenna arrays utilized by FD massive MIMO systems, that provide the ability of adaptive electronic beam control in both the elevation and azimuth dimensions. The main objective was to devise an elevation beamforming algorithm for a multi-cell FD massive MIMO system, utilizing the users' channel covariance matrices. We first derived a deterministic equivalent of the
SIR as a function of the downtilt antenna port weight vectors. The problem of finding the optimal downtilt vectors that maximize the minimum signal to intra-cell interference ratio in each cell while constraining the inter-cell interference leakage was then formulated and solved quasi-optimally using SDR and Dinkelbach's method. Simulations showed the superior performance of the SDB algorithm over the existing schemes.

\section{REFERENCES}

[1] F. Rusek, D. Persson et al., "Scaling up MIMO: Opportunities and challenges with very large arrays," IEEE Signal Processing Magazine, vol. 30, no. 1, pp. 40-60, Jan. 2013.

[2] 3GPP TR 36.897 V13.0.0 , "Study on elevation beamforming/FullDimension (FD) MIMO for LTE ," June 2015.

[3] 3GPP TR 37.840 V12.0.0, "Study of radio frequency (RF) and electromagnetic compatibility (EMC) requirements for active antenna array system (AAS) base station," Mar. 2013.

[4] K. Linehan and R. Chandrasekaran, "Active antennas: The next step in radio and antenna evolution," [Online]. Available: https://www.yumpu.com/en/document/view/8991085/ active-antennas-the-next-step-in-radio-and-antenna-evolution.

[5] Y. Kim, H. Ji, J. Lee, Y. H. Nam, B. L. Ng, I. Tzanidis, Y. Li, and J. Zhang, "Full dimension MIMO (FD-MIMO): the next evolution of MIMO in LTE systems," IEEE Wireless Communications, vol. 21, no. 2, pp. 26-33, April 2014.

[6] J. Koppenborg, H. Halbauer, S. Saur, and C. Hoek, "3D beamforming trials with an active antenna array," in ITG Workshop on Smart Antennas, 2012, pp. 110-114.

[7] G. X. et al., "Full dimension MIMO (FD-MIMO): Demonstrating commercial feasibility," IEEE Journal on Selected Areas in Communications, vol. 35, no. 8, pp. 1876-1886, Aug. 2017.

[8] N. Seifi, J. Zhang, R. W. Heath, T. Svensson, and M. Coldrey, "Coordinated $3 \mathrm{D}$ beamforming for interference management in cellular networks," IEEE Transactions on Wireless Communications, vol. 13, no. 10, pp. 5396-5410, Oct. 2014.

[9] W. L. et al., "Downlink vertical beamforming designs for active antenna systems," IEEE Transactions on Communications, vol. 62, no. 6, pp. 1897-1907, June 2014.

[10] B. Partov, D. J. Leith, and R. Razavi, "Utility fair optimization of antenna tilt angles in LTE networks," IEEE/ACM Transactions on Networking, vol. 23, no. 1, pp. 175-185, Feb. 2015.

[11] Radiocommunication Sector of International Telecommunication Union, "Report ITU-R M.2135-1: Guidelines for evaluation of radio interface technologies for IMT-advanced," 2009.

[12] I. Boukhedimi, A. Kammoun, and M. S. Alouini, "Coordinated SLNR based precoding in large-scale heterogeneous networks," IEEE Journal of Selected Topics in Signal Processing, vol. 11, no. 3, pp. 534-548, April 2017.

[13] M. Sadek, A. Tarighat, and A. H. Sayed, "A leakage-based precoding scheme for downlink multi-user MIMO channels," IEEE Transactions on Wireless Communications, vol. 6, no. 5, pp. 1711-1721, May 2007.

[14] Q. U. A. Nadeem, A. Kammoun, M. Debbah, and M. S. Alouini, "Spatial correlation characterization of a full dimension massive MIMO system," in 2016 IEEE Global Communications Conference (GLOBECOM), Dec. 2016, pp. 1-7.

[15] J. Nam, A. Adhikary, J. Y. Ahn, and G. Caire, "Joint spatial division and multiplexing: Opportunistic beamforming, user grouping and simplified downlink scheduling," IEEE Journal of Selected Topics in Signal Processing, vol. 8, no. 5, pp. 876-890, Oct. 2014

[16] R. Couillet and M. Debbah, Random matrix methods for wireless communications, 1st ed. New York, NY, USA: Cambridge University Press, 2011.

[17] E. Karipidis, N. D. Sidiropoulos, and Z. Q. Luo, "Quality of service and max-min fair transmit beamforming to multiple cochannel multicast groups," IEEE Transactions on Signal Processing, vol. 56, no. 3, pp. 1268-1279, March 2008.

[18] A. Zappone, L. Sanguinetti, G. Bacci, E. Jorswieck, and M. Debbah, "Energy-efficient power control: A look at 5G wireless technologies," IEEE Transactions on Signal Processing, vol. 64, no. 7, pp. 1668-1683, April 2016.

[19] K. Schäcke, "On the kronecker product," Masters Thesis, University of Waterloo, 2013. 